Bangladesh J. Bot. 49(1): 29-38, 2020 (March)

\title{
AGRONOMIC, PHYSIOLOGICAL AND MOLECULAR CHARACTERIZATION OF SPRING WHEAT (TRITICUM AESTIVUM L.) ACCESSIONS FOR DROUGHT TOLERANCE
}

\author{
Muhammad Qadir Ahmad, Muhammad Faroog Naseer, Abdul Qayyum, \\ Sami ul-Allah ${ }^{1}$, Waqas Malik, Sultan Habibullah Khan ${ }^{2}$, \\ Etrat Noor and Muhammad SaJjad ${ }^{3 *}$ \\ Department of Plant Breeding and Genetics, Faculty of Agricultural Sciences \\ and Technology, Bahauddin Zakariya University, Multan, Pakistan
}

Keywords: Spring wheat, Genetic diversity, Drought tolerance, ISSR, Molecular characterization

\begin{abstract}
A comprehensive study was conducted to investigate (i) the effect of drought stress on genetic association among various yield traits and (ii) molecular and phenotypic diversity in the selected spring wheat accessions. A panel of 24 spring wheat accessions was evaluated under normal irrigation and drought stress. Data were collected for chlorophyll contents, canopy temperature, days to heading, grain filling period, relative water content, plant height, peduncle length, number of kernels per spike, 1000-kernel weight and grain yield. Analysis of variance depicted significant differences for genotypes (G), effect of treatment (T) and interaction between $\mathrm{T}$ and $\mathrm{G}$. Correlation analysis revealed that genetic association among various traits was stronger in normal treatment than water stressed. The Biplot analysis grouped the genotypes into tolerant and susceptible groups. Molecular characterization of tolerant and susceptible genotypes was done with 14 ISSR markers. ISSR primers revealed a mean of 0.63 genetic similarities among genotypes. Polymorphism information content (PIC) values varied from 0.24 to 0.49 . The genetic diversity in selected germplasm can be used to develop drought tolerant lines considering the changing pattern of traits association under drought stress.
\end{abstract}

\section{Introduction}

Increasing world's population is expected to reach 9 billion by 2050 . It would intensify the environmental impacts by aggravating conflicts for water and land resources. Wheat being the most important grain crop will become very imperative to ensure food security under changing environmental conditions, increasing world's population and diminishing water reservoirs (Ain et al. 2015).

Due to insufficient rainfall and altered precipitation pattern, drought has become major threat to wheat production. Breeding for drought tolerant wheat cultivars is the ultimate mean to reduce the impacts of marginal precipitation and prolonged spells of dry weather (Ahmad et al. 2015). Drought tolerance is a multi-genic trait which implies a combination of physiological, biochemical and genetic mechanisms. Development of high yielding and drought tolerant cultivars demand sufficient time and capital. However, evaluation of already developed wheat genotypes against drought stress and high yield may serve as short term solution of the problem. Selection of genotypes based on yield related traits under drought stress is a suitable strategy to develop drought tolerant genotypes.

\footnotetext{
*Author for correspondence: <msajjadpbg@gmail.com>. ${ }^{1}$ College of Agriculture, BZU Bahadur Subcampus, Layyah, Pakistan. ${ }^{2}$ U.S. Pakistan Centre for Advanced Studies in Agriculture and Food Security, University of Agriculture Faisalabad, Pakistan. ${ }^{3}$ Department of Biosciences, COMSATS University Islamabad (CUI), Park Road, Islamabad 45550, Pakistan.
} 
Knowledge of plant traits associated with high yield under stressed condition is essential to explore the complex genetic mechanisms involved in wheat's adaptability (Sajjad et al. 2018). Grain yield is a quantitative trait which is controlled by many genes influenced by the environment. Wheat yield could be improved by focusing important yield components such as 1000-kernal weight and number of grains per plant (Ahmad et al. 2015). Drought affects various plant developmental stages such as heading, anthesis and grain filling (Hossain et al. 2013). Number of days to heading and days to grain filling would help in wheat's adaptability under terminal drought conditions (Lopes et al. 2012). Effect of drought stress on rate of photosynthesis could be determined by investigating spade value and canopy temperature. To determine the level of drought tolerance among genotypes it is necessary to examine their relative performance. Selection should target genotypes both under normal and stressed conditions for their good adaptation under environmental fluctuations.

Molecular characterization provides insight observation of the genetics of studied genotypes. Different molecular marker systems are being used for characterization of various crop plants. These systems vary in their principle, amount of polymorphism, cost and reproducibility (Ashraf et al. 2016). In wheat, Najaphy et al. (2012) revealed the efficiency of ISSR markers in accounting polymorphism and genetic profiling. Application of molecular markers for the recognition of variation in nucleotide sequences provides new insight measures of genetic diversity (Karkas et al. 2010). The present study aimed to identify (i) the extent of genetic variation among drought tolerant wheat genotypes and (2) association among various traits under optimum and drought stress.

\section{Materials and Methods}

The experimental material consisted of 23 wheat genotypes (Table 1) belonging to Syrian origin and one local variety Fareed-06 (drought tolerant) which were evaluated at the experimental area of the department of Plant Breeding and Genetics, Bahauddin Zakariya University, Multan, Pakistan.

The experiment was performed for two successive years 2013-14 and 2014-15, respectively. Genotypes were sown on $5 \mathrm{~m}$ plots containing two rows of each genotype. Row to row distance was maintained $30 \mathrm{~cm}$. Sowing was done with the help of hand driven drill in randomized complete block design (RCBD) in a set of three replications. The experiment was raised on 20 Nov. and 24 Nov. during 2013-14 and 2014-15, respectively. The genotypes were evaluated under two treatments e.g. irrigated and drought. Normal irrigated field received four irrigations (1st at tillering stage, 2nd at booting stage, 3rd at heading stage and 4th at grain filling stage). Drought stress was created by withholding irrigation after 1st irrigation; it was given to ensure better germination. The precipitation received in first year and second year was $19 \mathrm{~mm}$ and $38 \mathrm{~mm}$, respectively (http://www.pmd.gov.pk/).

Data were recorded on different agronomic and physiological traits from five randomly selected plants per replication viz., chlorophyll contents (SPAD) canopy temperature (CT), days to heading (DH), grain filling period (GFP), relative water content (RWC), plant height (PH), peduncle length (PL), number of kernels per spike (K/S), 1000-kernel weight (TKW) and grain yield per spike (GY/S). Leaf chlorophyll measurements were made on fully expanded flag leaves of main tiller during 10:00 a.m. to 12:00 p.m. using spad meter (SPAD-502).

Genomic DNA was obtained from 15 days old leaves using CTAB method (Muray and Thompson 1980). of DNA extraction with minor modifications as suggested by Khan et al. (2004). Fourteen ISSR primers were used to characterize 24 wheat genotypes. For PCR analysis stock DNA was diluted to $30 \mathrm{ng} / \mathrm{ul}$ conc. PCR analyses was performed according to Sajjad et al. 
(2014). The PCR products were separated using $2 \%$ agarose gel electrophoresis in $1 \mathrm{X}$ TBE buffer. The gel was stained with ethidium bromide. Initially gel was visualized on trans illuminator and finally was documented using gel documentation system (Photonyx, USA).

Table 1. List of wheat genotypes and their selection history.

\begin{tabular}{|c|c|}
\hline Code & Selection history \\
\hline 01 & ND/VG9144//KAL/BB/3/YACO/4/VEE\#5 \\
\hline 02 & AO41/EMU`S`/TEVEE`S`/3/SD 8036 \\
\hline 03 & TURACO/CHIL/6/SERI 82/5/ALD`S`/4/BB/GLL//CNO67/7C/3/KVZ/TI \\
\hline 04 & BOCRO-4/3/MAYON`S'//CROW'S`/VEE`S` \\
\hline 05 & VAN`S`/3/CNDR`S`/ANA//CNDR`S`/MUS`S`/4/TEVEE-5 \\
\hline 06 & SHUHA-8/DUCULA \\
\hline 07 & CHAM-4/SHUHA`S $/ 6 / 2 / * S A K E R / 5 / R B S / A N Z A / 3 /$ \\
\hline 08 & PAVON 76 \\
\hline 09 & CHAM-4/SHUHA`S`/6/2/*SAKER/5//TOB/4/BOW`S` \\
\hline 10 & NS732/HER//ARRIHANE/3/PGO/SERI//BAU \\
\hline 11 & IZAZ-2//TEVEE`S`SHUHA`S` \\
\hline 12 & GIZA-168//SHUHA`S`/DOBUC'S` \\
\hline 13 & FERROUG-2/POTAM*2KS8112161-8//ZEMAMRA-8 \\
\hline 14 & SERI 82/SHUHA`S //DOVIN-2 \\
\hline 15 & SOMAMA-9//SERI 82SHUHA`S` \\
\hline 16 & SHUHA-7/4/NIF/3/SOTY//NAD63/CHRIS \\
\hline 17 & ACHTAR*3//KANZ/KS85-8-4/3MON`S'/ALD'S`//BOW'S` \\
\hline 18 & TEVEE`S`/3/T.AEST/SPRW`S`/CA8055/4/PASTOR-2/5/SUNBRI \\
\hline 19 & FRET-2/2*PASTOR-2 \\
\hline 20 & ZEMAMRA-1/SOMAMA-3 \\
\hline 21 & HUBARA-5/3/SHA3/SERI//SHA4/LIRA \\
\hline 22 & HUBARA-3/ANGI-2//SOMAMA-3 \\
\hline 23 & CROC-1/AE.SQUARROSA(224)//OPATA/3/QAFZAH-1/4/SOMAMA-3 \\
\hline 24 & Fareed-06 \\
\hline
\end{tabular}

The agronomic and physiological traits were analyzed for analysis of variance (Steel et al. 1997), correlation coefficient analysis using MSTATC software. Principal component analysis was performed as suggested by Ahmad et al. (2014). For molecular analysis; ISSR data was scored for each band on the basis of presence (1) and absence (0) to generate binary data matrix. NTSYSpc 2.0 software was used to construct dendrogram on the basis of Jacard,s distance matrix using Unweighted Pair Group Method of Arithmetic Means (UPGMA). Polymorphism information content (PIC) value of each primer was calculated as described by Ashraf et al. (2016). Other indices which were calculated include marker index (MI), confusion probability (cj), and discriminating power $(\mathrm{Dj})$ (Anderson et al. 1993).

\section{Results and Discussion}

All the traits were negatively affected by drought stress. Wheat genotypes differed significantly for SPAD, CT, DH, GFP, RWC, PH, PL, K/S, TKW and GY/S (Table 2). Treatment effect (drought) showed significant influence on all the traits. Year factor did not affect all the traits, only DH, CT, RWC and GY/S were influenced over the years whereas, other traits depicted non-significant results. Interaction between wheat genotypes and stress treatment were also observed significant (Table 2). 


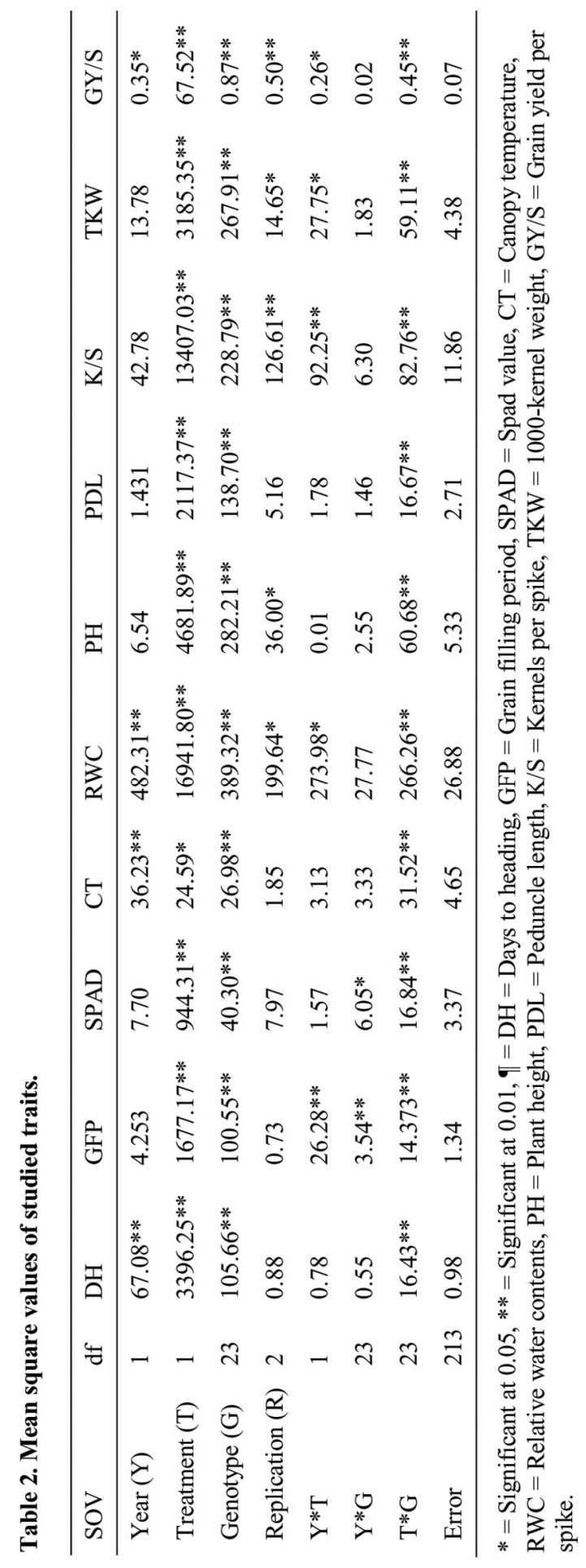


Table 3 shows the mean values of all the traits recorded under normal and stress conditions over both years. Average DH under normal conditions was observed 97.58 and 98.45 for both years, respectively whereas minimum days to heading were taken by the genotype (HUBARA) and maximum days were recorded for the genotype (CHAM-4). Similarly under drought conditions DH means were observed 90.5 and 91.6 days, respectively. Under stress condition minimum days to heading (84.7) were taken by the genotype (IZAZ-2 and HUBARA) and maximum days (95) were taken by the genotype (NS732 and SHUHA). Performance of the check cultivar (Fareed-06) for DH was observed (91) days under normal conditions and (86) days under drought condition. Average grain yield per plant was observed $2.78 \mathrm{~g}$ under normal irrigation over both years whereas under stressed environment slight fluctuation in average grain yield (1.74-1.88 g) was observed over both years. All the traits showed reduction in trait values under stress condition except canopy temperature where increase in trait values was observed. Under normal conditions CT value was found to range from (18.7 - 30.7) with average value (24.34) whereas under drought environment it was observed form (19.5 - 28.67) with average (24.9).

Table 3. Average, minimum and maximum values of all traits $\mathbb{I}$ studied under both conditions.

\begin{tabular}{lccccccc}
\hline Trait & & \multicolumn{3}{c}{ Year 1 } & & \multicolumn{3}{c}{ Year 2 } & \\
\cline { 3 - 7 } & & Ave & Min. & Max. & Ave. & Min. & Max. \\
\hline DH & N & 97.58 & 89.67 & 102.33 & 98.45 & 90.67 & 103.00 \\
& D & 90.52 & 84.67 & 94.33 & 91.60 & 86.00 & 95.00 \\
GFP & N & 28.87 & 23.3 & 35.67 & 29.78 & 22.30 & 36.67 \\
& D & 24.60 & 19.00 & 29.30 & 24.21 & 19.00 & 28.30 \\
SPAD & N & 48.13 & 44.17 & 50.17 & 47.70 & 41.33 & 50.63 \\
& D & 44.30 & 38.27 & 48.67 & 44.12 & 36.40 & 48.63 \\
CT & N & 24.80 & 20.07 & 30.77 & 23.89 & 18.73 & 30.30 \\
& D & 25.20 & 24.15 & 26.20 & 24.65 & 19.53 & 28.67 \\
RWC & N & 66.85 & 50.10 & 83.10 & 66.22 & 49.97 & 81.43 \\
& D & 53.74 & 45.37 & 64.67 & 48.83 & 42.67 & 66.67 \\
PH & $\mathrm{N}$ & 85.65 & 76.63 & 96.80 & 85.99 & 76.17 & 97.50 \\
& $\mathrm{D}$ & 77.46 & 67.33 & 86.00 & 77.84 & 68.33 & 87.33 \\
PDL & $\mathrm{N}$ & 29.86 & 24.00 & 35.13 & 30.21 & 24.00 & 35.33 \\
& $\mathrm{D}$ & 24.66 & 16.30 & 30.86 & 24.53 & 18.67 & 30.10 \\
K/S & $\mathrm{N}$ & 54.97 & 46.33 & 65.00 & 54.58 & 45.00 & 65.00 \\
& $\mathrm{D}$ & 40.04 & 28.33 & 51.67 & 42.00 & 29.33 & 49.33 \\
TKW & $\mathrm{N}$ & 40.84 & 31.43 & 55.27 & 40.70 & 30.70 & 54.17 \\
& $\mathrm{D}$ & 33.45 & 23.87 & 46.20 & 34.58 & 26.60 & 46.57 \\
GY /S & $\mathrm{N}$ & 2.78 & 2.07 & 3.47 & 2.78 & 2.10 & 3.47 \\
& $\mathrm{D}$ & 1.74 & 1.15 & 2.43 & 1.88 & 1.20 & 2.57 \\
\hline
\end{tabular}

Correlation analysis showed significant positive association of DH with SPAD, CT, RWC, $\mathrm{K} / \mathrm{S}$ and significant negative association with GFP under normal conditions, whereas under drought conditions DH showed significant negative associations with GFP, CT, TKW. Grain filling period showed significant negative correlation with SPAD, CT, RWC, K/S, GY/S under normal condition whereas under drought stress it showed positive association with TKW and GY/S. All the associations of GFP under normal conditions were negative whereas under drought observed positive. Degree of correlation among other agronomic traits is presented in Table 5. PCA biplot grouped the genotype into two main groups a susceptible group and tolerant group of genotypes. Some genotypes were appeared more tolerant to local check. Highest trait vector was 
observed for PDL followed by PH and GY/S which showed these traits were more influenced by drought treatment (Fig. 1)

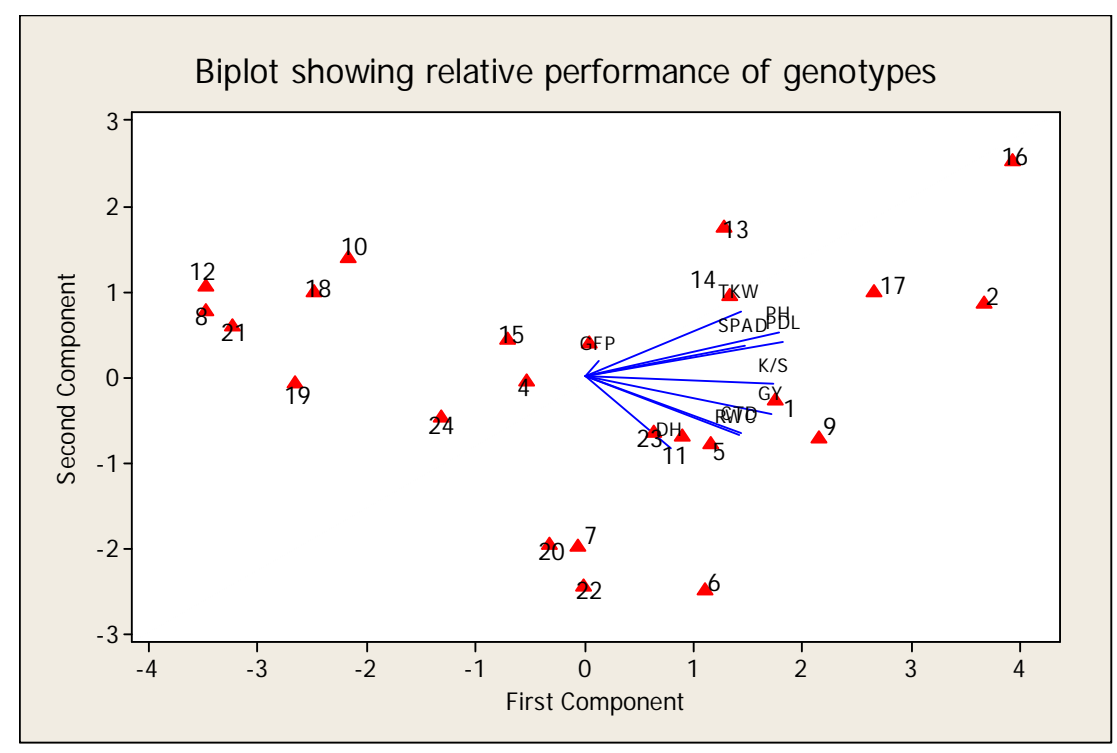

Fig. 1. Principal component biplot based on relative performance.

The 14 ISSR primers applied to study 24 wheat genotypes generated $4-7$ bands with an average 5 bands per primer (Table 4). A total of 70 bands were generated, among them 48 (68.6\%) were polymorphic and $22(31.4 \%)$ were monomorphic. The primer UBC-818, UBC-826 and UBC-835 showed highest percentage of polymorphism (100\%) whereas lowest percentage of polymorphism (20) was revealed by (primer 8). Polymorphism information content values varied from 0.24 to 0.49 with an average of 0.39 . In this investigation, MI values ranged from $0.41-2.1$. Lowest value for MI was revealed by UBC- 845 whereas highest value was accounted by UBC827. Values of RP, CJ and DJ are represented in Table 5.

UPGMA based cluster analyses was executed to generate a dendrogram. ISSR primers revealed a mean of 0.63 genetic similarities among 24 wheat genotypes. Similarly values were found to range from 0.41 to 0.82 (Fig. 2). The dendrogram grouped genotypes into 3 main clusters A, B and C. Cluster A contained 4 genotypes i.e. (HUBARA-5, HUBARA-3, CROC-1, and Fareed-06). Cluster B was further divided into 3 sub-clusters 1B, 2B and 3B. Sub-cluster 1B consisted of 3 genotypes i.e. (TURACO, BOCRO-4 and VAN'S'). Similarly, sub-cluster 2B consisted of 4 genotypes (CHAM-4, PAVON 76, CHAM-4 and NS732). Sub-cluster 3B contained 5 genotypes (AO41, VAN'S, IZAZ-2, GIZA-168, FERROUG-2). Cluster 3 has 2 sub-clusters 1C and 2C. Sub-cluster 1C comprised of 5 genotypes (SERI 82, ACHTAR*3, SOMAMA-9, TEVEE`S, SHUHA-7) whereas 2C consisted of only 2 genotypes (ND/VG9144 and FRET-2). Maximum similarity (0.82) was observed between the genotype (SOMAMA-9 and TEVEE'S) located in sub-cluster $1 \mathrm{C}$ whereas least similarity was observed between the genotypes (CROC-1 and PAVON 76) located in same sub-cluster.

Grain yield is governed by many morpho-physiological traits and greatly influenced by limited water supply which reduces the translocation of photosynthates to developing kernels (Sajjad et al. 2014). Selections for high yielding genotypes under optimum conditions allow 
genotypes to maintain their high ranks in stress environment, because it is expected that the same genotypes will perform high under stressed environments (Mwadzingeni et al. 2016). Here, reduction in grain yield was observed in all genotypes under drought stress. But some genotypes maintained their ranks both under optimum and stressed conditions. The genotypes which showed less reduction in their yield under drought conditions include (HUBARA-5, TEVEE'S\%, NS732) may be considered as tolerant genotypes; contrarily to sensitive genotypes which showed more reduction in their yield include (AO41, VAN'S and CHAM-4) (Mwadzingeni et al. 2016).

Table 4. Marker information.

\begin{tabular}{|c|c|c|c|c|c|c|c|c|c|}
\hline $\begin{array}{l}\text { Sl. } \\
\text { No }\end{array}$ & $\begin{array}{l}\text { Marker } \\
\text { name }\end{array}$ & $\begin{array}{l}\text { Average } \\
\text { PIC }\end{array}$ & $\begin{array}{l}\text { No. of } \\
\text { bands }\end{array}$ & $\begin{array}{l}\text { Poly- } \\
\text { morphic }\end{array}$ & $\begin{array}{l}\% \text { poly- } \\
\text { morphism }\end{array}$ & MI & $\mathrm{RP}$ & $\begin{array}{l}\text { Av. } \\
\text { CJ }\end{array}$ & $\begin{array}{l}\mathrm{Av} . \\
\mathrm{Dj}\end{array}$ \\
\hline 1 & UBC-807 & 0.25 & 5 & 2 & 40.00 & 0.497 & 0.583 & 0.741 & 0.630 \\
\hline 2 & UBC-810 & 0.46 & 5 & 4 & 80.00 & 1.854 & 3.167 & 0.516 & 0.742 \\
\hline 3 & UBC-812 & 0.39 & 7 & 5 & 71.43 & 1.955 & 2.917 & 0.592 & 0.704 \\
\hline 4 & UBC-813 & 0.48 & 4 & 2 & 50.00 & 0.965 & 1.667 & 0.496 & 0.752 \\
\hline 5 & UBC-814 & 0.47 & 5 & 1 & 20.00 & 0.469 & 0.750 & 0.511 & 0.745 \\
\hline 6 & UBC-815 & 0.27 & 6 & 5 & 83.33 & 1.348 & 2.000 & 0.719 & 0.641 \\
\hline 7 & UBC-818 & 0.39 & 5 & 5 & 100.00 & 1.944 & 2.833 & 0.594 & 0.703 \\
\hline 8 & UBC-825 & 0.28 & 7 & 3 & 42.86 & 0.826 & 1.000 & 0.713 & 0.644 \\
\hline 9 & UBC-826 & 0.24 & 5 & 5 & 100.00 & 1.207 & 1.083 & 0.799 & 0.600 \\
\hline 10 & UBC-827 & 0.42 & 7 & 5 & 71.43 & 2.118 & 3.167 & 0.558 & 0.721 \\
\hline 11 & UBC-828 & 0.45 & 5 & 4 & 80.00 & 1.813 & 2.833 & 0.527 & 0.736 \\
\hline 12 & UBC-835 & 0.49 & 4 & 4 & 100.00 & 1.972 & 3.667 & 0.486 & 0.757 \\
\hline 13 & UBC-845 & 0.41 & 3 & 1 & 33.33 & 0.413 & 0.583 & 0.569 & 0.716 \\
\hline 14 & UBC-846 & 0.27 & 7 & 4 & 57.14 & 1.090 & 1.333 & 0.716 & 0.642 \\
\hline Total & ----- & ----- & 70.00 & 48.00 & ---- & ---- & ---- & ---- & ---- \\
\hline Means & & 0.39 & 5.38 & 3.69 & 68.42 & 1.38 & 2.08 & 0.60 & 0.70 \\
\hline
\end{tabular}

DH showed negative association with GFP under both conditions. It could be explained that if a genotype starts heading earlier; will have more time for grain filling. Contrarily, late heading genotypes tends to decrease their GFP due to increased temperature near to maturity (Al-Karaki 2012). Here positive correlation between DH and SPAD and RWC could be explained as these genotypes contain less chlorophyll contents, less water contents and therefore started heading earlier. DH showed negative association with TKW under drought condition, reason may be that early heading genotypes have more GFP which resulted in increased TKW. TKW and GY/S were also associated positively. It could be a reason that increased GFP resulted in more time for formation and translocation of photosynthates to developing grains which ultimately resulted in increased TKW and yield (Guendouz and Maamari 2012).

Negative association between GFP and SPAD suggested that high concentration of chlorophyll effects GFP negatively. As an interesting fact of the study the genotypes which showed low CT tend to heading early and GFP late. Hamblin et al. (2014) explained that reduced chlorophyll contents minimize heat load and water requirements of the plant which resulted in cool leaves. Therefore, it will be justified to say that low SPAD value resulted in cooler plants which resulted in more grain filling duration.

Molecular characterization was carried out to depict the genetic diversity in studied plant material for drought tolerance and to strengthen the classification based on morphological characterization. In the present study, primer UBC-835 showed high PIC value coupled with high $\mathrm{Dj}$ and low $\mathrm{Cj}$ revealed the ability of this primer in assessment of genetic variation. High MI value of ISSR -3, high PIC, and Dj showed distinctive nature of this primer (Ashraf et al. 2016). 


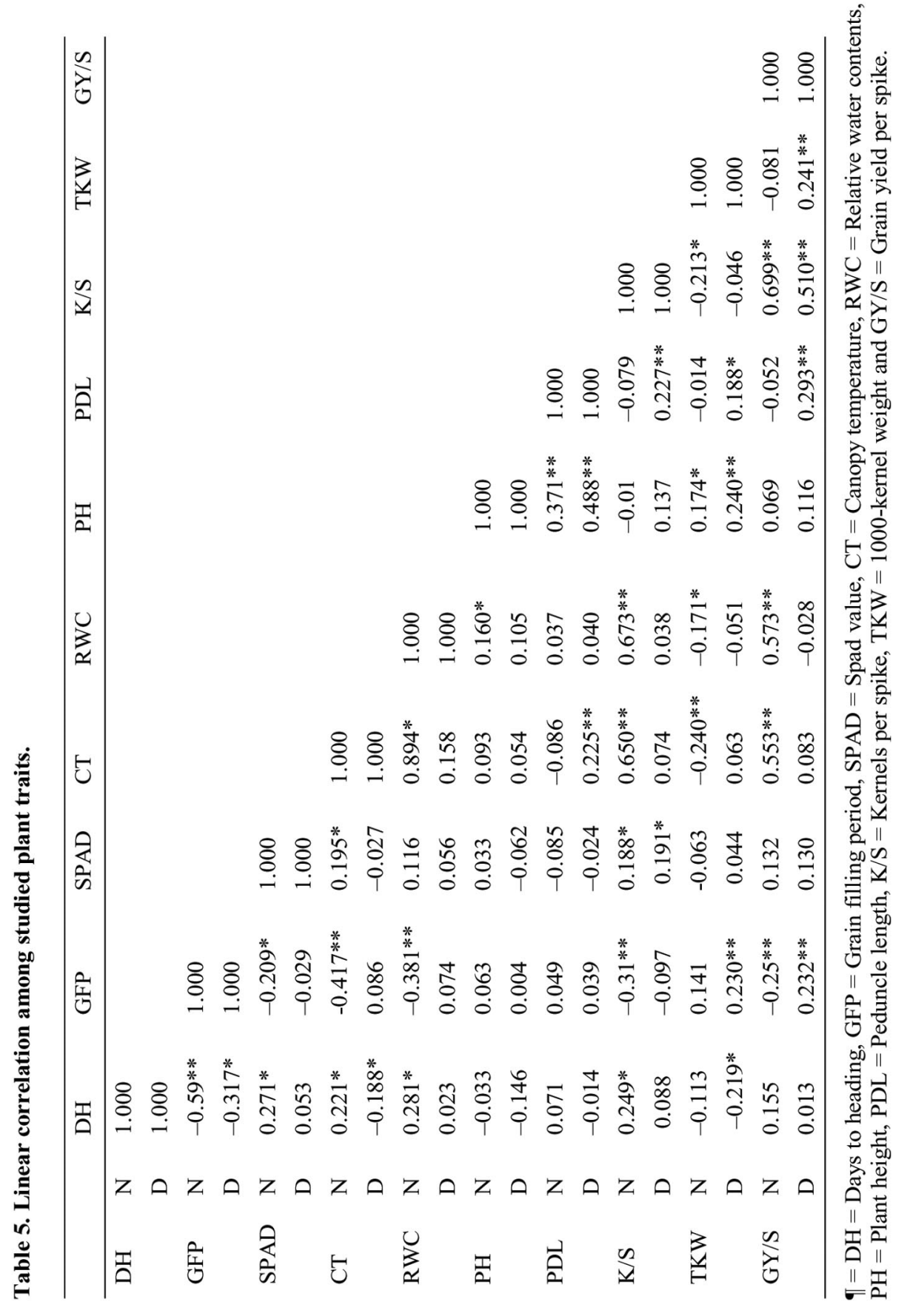


Dendrogram generated on the basis of molecular characterization revealed the grouping of genotypes on the basis of genetic similarity. Grouping based on morphological characterization (Fig. 1) and grouping based on molecular characterization (Fig. 2) represented very interesting results. Molecular results supported morphological results in most of the cases. The genotypes (TEVEE'S) and (SOMAMA-9) showed highest level of similarity (0.82) whereas; same genotypes were also located very close to each other on biplot ordination (Fig. 1). Both genotypes appeared

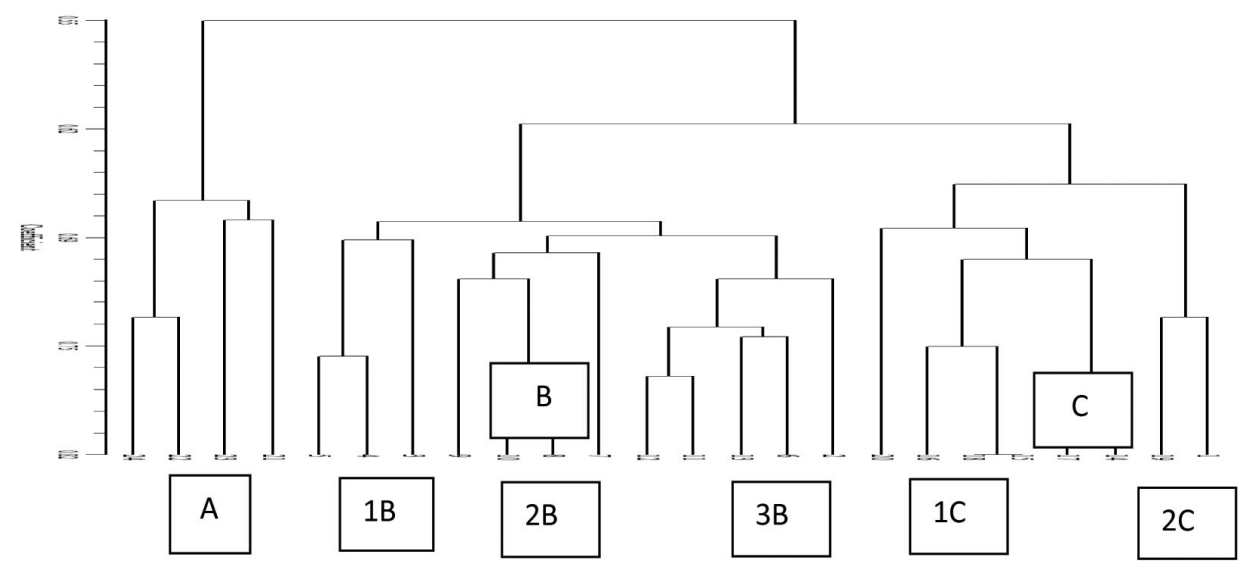

Fig. 2. Dendrogram generated on the basis of molecular characterization.

as tolerant genotypes. Similarly, the genotypes (PAVON 76) and (GIZA-168) expressed (0.75) similarity and both were located on very close on biplot in tolerant group. Both genotypes appeared as tolerant genotypes therefore, there similar tolerant behavior under drought condition is attributed to their genetic similarity. Contrarily, the genotypes (PAVON 76) and (CROC-1) showed lowest level of similarity. Similar grouping of genotypes by both morphological traits and ISSR markers revealed the authenticity of the markers. Thus, use of ISSR markers can save labor and time and environment barriers for efficient characterization of drought tolerance in wheat.

\section{References}

Ahmad MQ, Khan SH, Sajjad M and Khan IA 2015. Analysis of drought responsive traits in hexaploid wheat (Triticum aestivum L.). Pak. J. Agri. Sci. 52: 701-707.

Ain Q, Rasheed A, Anwar A, Mahmood T, Imtiaz M, Mahmood T, Xia X, He Z and Quraishi UM 2015. Genome-wide association for grain yield under rainfed conditions in historical wheat cultivars from Pakistan. Front. Plant Sci. 6: 743. doi: 10.3389/fpls.2015.00743.

Al-Karaki GN 2012. Phenological development-yield relationships in durum wheat cultivars under lateseason high-temperature stress in a semiarid environment. I.S.R.N. Agron. doi: 10.5402/2012/456856.

Ashraf J, Malik W, Iqbal MZ, Khan AA, Qayyum A, Noor E, Abid MA, Cheema HMN and Ahmad MQ 2016. Comparative analysis of genetic diversity among Bt cotton genotypes using EST-SSR, ISSR and morphological markers. J. Agr. Sci. Tech. 18: 517-531.

Anderson JA, Churchill GA, Autrique JE, S.D. Tanksley and Sorrells ME 1993. Optimizing parental selection for genetic linkage maps. Genome 36: 181-186.

Guendouz A and Maamari K 2012. Grain-filling, chlorophyll content in relation with grain yield component of durum wheat in a mediterranean environment. Afr. Crop Sci. J. 20: 31-37. 
Hamblin J, Stefanova K and Angessa TT 2014. Variation in chlorophyll content per unit leaf area in spring wheat and implications for selection in segregating material. PLoS ONE 9: e92529 doi.org/10.1371/journal.pone.0092529

Hossain A, Sarker M, Saifuzzaman M, Teixeira da Silva J, Lozovskaya M and Akhter M 2013. Evaluation of growth, yield, relative performance e and heat susceptibility of eight wheat (Triticum aestivum L.) genotypes grown under heat stress. Int. J. Plant Prod. 7: 615-636.

Karakas SO, Gurel F and Uncuoglu AA 2010. Exploiting a wheat EST database to assess genetic diversity. Genet. Mol. Biol. 33: 719-730.

Khan IA, Awan FS, Ahmad A and Khan AA 2004. A modified mini prep. method for economical and rapid extraction of genomic DNA in plants. Plant Mol. Biol. Repor. 22: 89a-89e.

Lopes M, Reynolds M, Jalal-Kamali M, Moussa M, Feltaous Y and Tahir I 2012. The yield correlations of selectable physiological traits in a population of advanced spring wheat lines grown in warm and drought environments. Field Crops Res. 128: 129-136.

Murray MG. and Thompson WF. 1980. Rapid isolation of high molecular weight plant DNA. Nucl. Acids Res. 8: 4321-4326.

Mwadzingeni L, Shimelis H, Tesfay S and Tsilo TJ 2016. Screening of bread wheat genotypes for drought tolerance using phenotypic and proline analyses. Front. Plant Sci. 7:1276. doi: 10.3389/fpls.2016.01276

Najaphy A, Parchin RA and Farshadfar B 2012. Comparison of phenotypic and molecular characterizations of some important wheat cultivars and advanced breeding lines. Aus. J. Crop Sci. 6: 326-332.

Sajjad M, Khan SH., Ahmad MQ, Rasheed M, Mujeeb-Kazi A and Khan I 2014. Association mapping identifies QTLS on wheat chromosome 3A for yield related traits. Cer. Res. Commun. 42: 177-188.

Sajjad M, Khan SH and Shahzad M 2018. Patterns of allelic diversity in spring wheat populations by SSR markers. Cyt. Genet. 52(2): 155-160.

Steel RGD, Dickey DA and Torrie JH 1997. Principles and procedures of statistics: A biometrical approach. McGraw-Hill Co. New York.

(Manuscript received on 17 July, 2018; revised on 10 December, 2018) 